\title{
Electrical Properties of Granular Silicon
}

\author{
L.O. Olimov*, Z.M. Sokhibova \\ Andijan Machine Building Institute, Russia. \\ *Corresponding Author: L.O. Olimov, Andijan Machine Building Institute, Uzbekistan.

\begin{abstract}
In the work, the electrical properties of granular silicon in the temperature range of $\sim 20 \div 450{ }^{\circ} \mathrm{C}$ were experimentally investigated. It is show that the temperature dependence of conductivity is associated with the location of the granule. The proposed considerations and the results obtained are of significant interest in the study of micro and nanocrystalline semiconductors.
\end{abstract}

Keywords: structura, specific resistance, semiconductor, potential barrier, move levels, localized traps, granula, conductivity of the traps, charge transfer.

\section{INTRODUCTION}

Within the framework of representations developed in the first part of our work [1], we suggested simple answer to the issue what the mechanism of charge transfer in granular semiconductors is. It was believe that the processes of charge transfer in granular semiconductors depend on the size, structure and location of the granules. That is, the location of the granules can be in two forms, e.g., in series or in parallel next to each other. Accordingly, the transfer process of charge carriers in them is fundamentally different. In the first variant, i.e., if the granules are arrange in series, the transfer of the charge from the first granule to the second granule will occur through the two contacting regions. In this case, the increase in trapped charge on localized traps in the two contacting regions leads to an increase in the height of the potential barrier, and this simultaneously leads to an increase in the resistivity. To the second variant, if, the granules are arrange parallel to each other, the contacting area, also forms parallel between the granules as in the first variant, local energy levels are formed in it. However, in this case, the charge carriers do not move from one granule to the other, they are trapped in traps ( $E_{i}$ levels), that are parallel to the formed contacting regions, and move to $E_{i}$ levels. Wherein, there is an increase in the total conductivity of the traps $\left(Y_{s s}\right)$. In this case, the overall specific conductivity is determined by the total specific conductivities. In this connection, in the present work, we discuss the experimental results of the charge transfer process in granular silicon, and the proposed mechanism is specifies.

\section{RESEARCH METHOD}

As mentioned in the previous article [1], there are a number of methods for obtaining granular semiconductor materials, of which the powder technology method is a promising method for producing polycrystalline semiconductor materials for solar cells or integrated circuits, as well as for thermoelements. Silicon granules were obtained in the framework of powder technology, which are given in work [ $2 \div 5]$, and have been used by us for obtaining a polycrystalline silicon plate. As a raw material of the technology, single-crystal silicon substrates were use, where solar cells with a coefficient of about $15 \%$ are use on their basis. The starting samples are ground in a ball mill, wherein the powder can obtain with a granules size of ten to a thousand nanometers controlling the grinding time. In order to research structure of the granular silicon electronic microscope was used and to assess the transfer of charge carriers the specific resistance $(\rho)$ of the granules was measured by two-probe method in a temperature range of 20 to $300{ }^{\circ} \mathrm{C}$ [4]. The measurements are conduct in semi-automatic mode directly with the granules heated, as in a temperature rise from 20 to $300^{\circ} \mathrm{C}$, and at the stage of its decrease.

\section{RESULTS AND DiSCUSSION}

As mentioned above and in the previous article [1], the granules can arranged in two variants, in series or parallel to each other. First, let us see the first variant.

International Journal of Research Studies in Electrical and Electronics Engineering (IJRSEEE) Page | 1 
Variant 1. Sequential location of granules. (Fig. 2). Usually such structures are observe larger sizes, for example, in granules with sizes of $100 \div 1000$ micrometers. Figure 1 illustrates the temperature dependence of the specific resistance $(\rho)$ of granular silicon, $(1-1000 \mu \mathrm{m}, 2-300 \mu \mathrm{m}$ and $3-100 \mu \mathrm{m})$. From Fig. 1, it can see that the specific resistance in all cases increases with temperature. With decreasing granules size $(1 \rightarrow 2 \rightarrow 3)$, the growth in specific resistance is observed more strongly than in others.

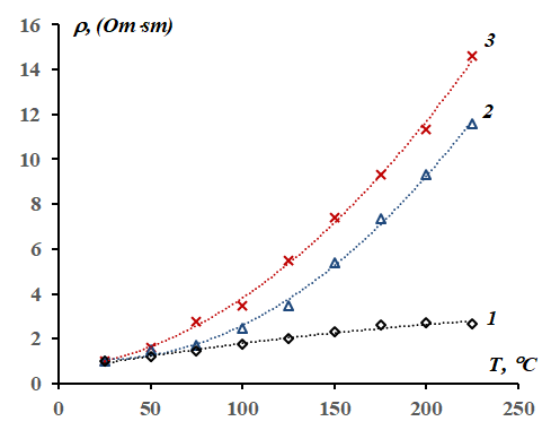

Fig1. Dependence of the specific resistance on temperature.

From the proposed mechanism for the emergence of carrier-charge transfer in granular semiconductors, in the first part of our work [1] it is known that the specific resistance is described by the expression:

$\rho=\frac{k}{q\langle a\rangle A^{*} T} \exp \left(q \varphi / k T^{)}\right.$

where $\langle a\rangle$ is the grain size, $\varphi$ is the potential barrier height on the grain boundary.

The potential barrier height

$\varphi=\frac{Q_{i}^{2}}{8 \varepsilon \varepsilon_{o} q N_{G}}$

Here, $Q_{i}$ is the boundary derivative charge in the grain boundaries, $N_{G}$ - is the concentration of an electrically active dopants, $\varepsilon_{o}$ and $\varepsilon$ - dielectric constant.

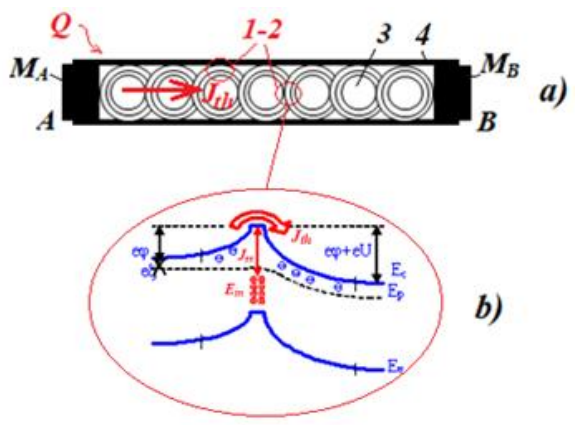

Fig2. Simplified scheme of location of the granule $(a)$, zone diagram $(b) .(1,2,3)$ - silicon granules, 4 - ceramic tube, $M_{A}$ and $M_{V}$ ohmic contacts respectively, in the $A$ and $B$ regions.

It is seen from (1) that an increase in the trapped charge $Q_{i}$ on localized traps in the boundaries between the granules, that is, in the two contacting regions of the granule, leads to an increase in $\varphi$, and this simultaneously leads to an increase in $\rho$ (Fig. 2a). An equivalent electrical scheme of such a structure is presented on fig.2. It can be seen that the equivalent electrical scheme consists of the series-connected resistances of the granule and the two contacting regions of the granule. In this case, the total specific resistance is determined by the total specific resistances. In addition, it can be seen from (1) that the specific resistance is related to the granule size $\langle a\rangle$, that is, a decrease in the granule size $\langle a\rangle$ leads to an increase in $\rho$. From Fig. 1 , it is seen that with a decrease in the size of the granule $(1 \rightarrow 2 \rightarrow 3)$, the growth of resistivity is observed more strongly than in others. This indicates that an increase in the trapped charge $Q_{i}$ on localized traps in the boundaries between the granules, in smaller sizes is observed more strongly. 
Variant 2. Arrangements of granule next to each other (in Fig. 6). Usually such structures are observed in smaller sizes, for example, in granules with dimensions of $400 \div 700$ micrometers. Figure 3 illustrates the temperature dependence of the specific conductivity $(\sigma)$ of granular silicon, it is very different from the first. From Fig. 3, it can be seen that the specific conductivity and the dark current increases with temperature. (Fig. 4). It also shows a decrease in voltage and growth (Figure 5). To explain the results, we use the model of thermionic emission [4:7] with additions concerning the account of the currents arising in the process of capture and emission of charge carriers in traps, which we formulated in [1], and also by a simplified sample scheme (Figure 6a) and the zone diagram between the charged granules (Fig. 6b).

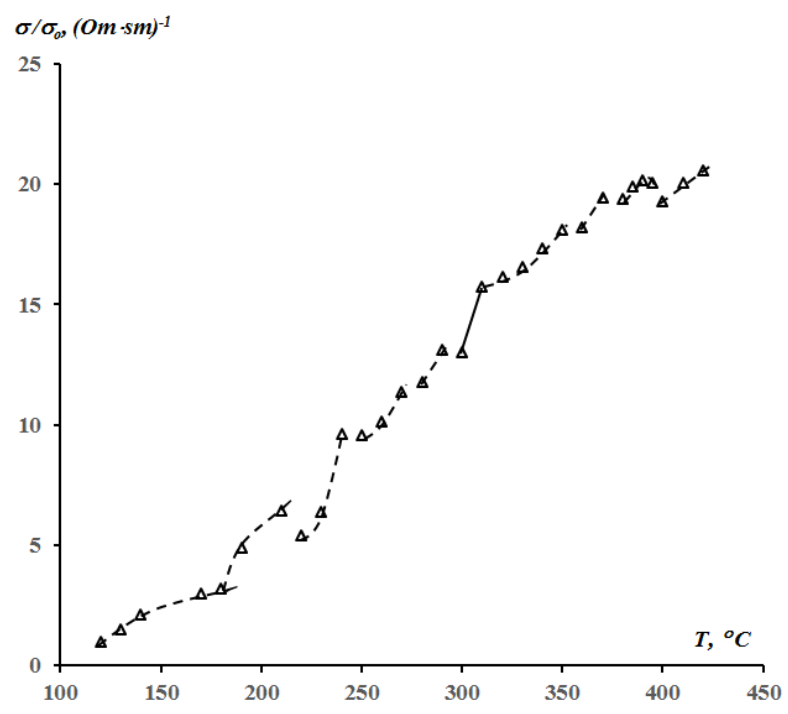

Fig3. Dependence of the conductivity on temperature.

It is known that for semiconductors the decrease in the resistivity is associated with the onset of intrinsic conductivity with increasing temperature. In addition, this increases the concentration of charge carriers and this in turn leads to an increase in current. The result of the research confirms this mechanism. However, according to the location of the granules, as described in the first part of our work [1] (Fig. $5)$, the charge transfer process must occur in two connected regions.

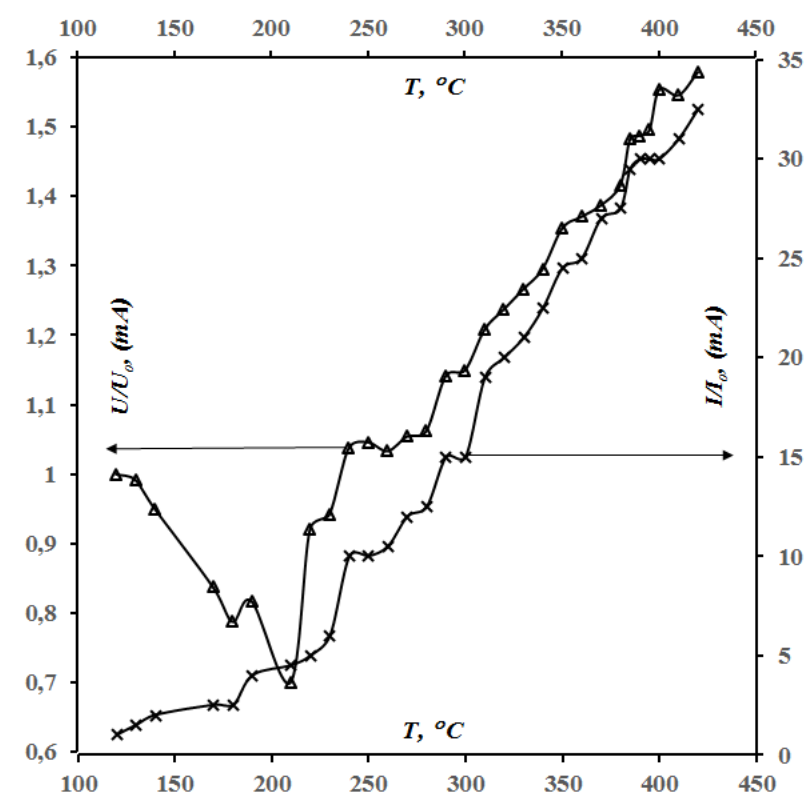

Fig4.Dependence of dark current and of voltage on temperature.

From Fig. 5b, it follows that charge carriers are capture by states $\left(E_{i}\right)$ at the boundary region. Emerged charge is compensate by ionized charges located in the space charge region. During the capture and emission of charge carriers on traps observed $J_{s s}$ currents arise and they move along $E_{i}$ levels located in region 5 (Figure 5). The current $J_{s s}$ is: 


$$
J_{s S}=Y_{s s} \delta \varphi
$$

where $Y_{s s}$ is the characteristic total conductivity of the traps, which depends both on their capture cross section and on the energy distribution, as well as on the position in space, that is, the location in region 5 (Fig. 5), $\delta \varphi$ is the change in the height of the potential barrier.

The current $J_{s s}$ is identically equal to the derivative in time with respect to charge bound on the boundary surface. In region, 5 (Fig. 5) the following situation takes place [4, 6, 8]: to the process of whole capture and emission on the boundary surface, the width of the spatial charge region should vary in order for total electroneutrality to be kept. This, in turn, influences the whole zone diagram (Fig. 5b), i.e., as a change in the barrier height $\delta \varphi$, so and on $Y_{s s}$. This means that the current $J_{s s}$ and the change in the barrier height $\delta \varphi$ are interrelated, and the vibrational properties of this interrelation are determined by the properties of the traps. Naturally, in the process of temperature change, both capture and emission of charge carriers with the participation of traps are observe. When the number of charge carrier retention is greater than their emission, the motion of the charge along the levels of the traps along the boundary of the two contacting granules (in region 5, Fig. 5a) is accompanied by a growth in the total conductivity of the traps. If the emission prevails over the capture, then the conductivity of the traps also increases, despite the fact that the charge moves in the opposite direction.

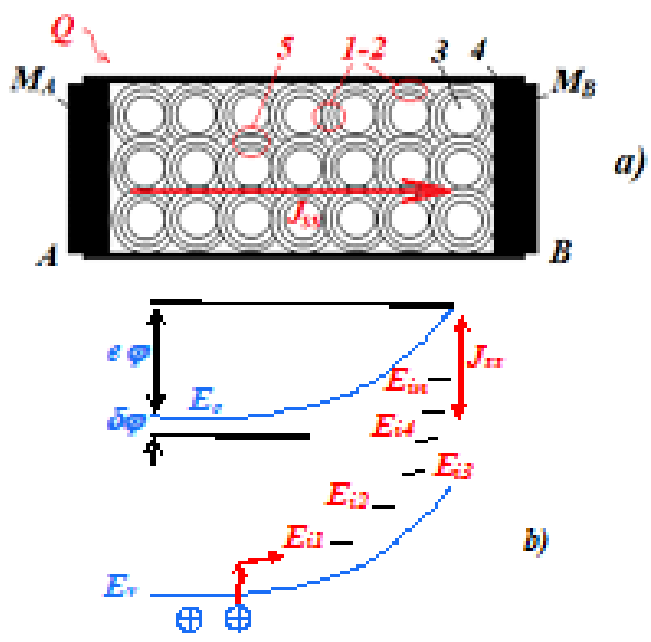

Fig5. Simplified scheme of location of the granule (a), zone diagram (b). (1, 2, 3) - silicon granules, 4 - ceramic tube, $M_{A}$ and $M_{B}$ contacts, respectively, in the $A$ and $B$ regions.

In this work $[4,6,8]$, we studied the manifestations of the energy states conditioned by traps, for example, with $E_{i 1} \sim 0,15 \mathrm{eV}, E_{i 2} \sim 0,17 \mathrm{eV}, E_{i 3} \sim 0,36 \mathrm{eV}, E_{i 4} \sim 0,3 \mathrm{eV}$ which are manifested at temperatures $\mathrm{T} \sim 70 \div 420{ }^{\circ} \mathrm{C}$. Current changes for example, growth at $\mathrm{T} \leq 150{ }^{\circ} \mathrm{C}$, $\mathrm{T} \sim 180 \div 230{ }^{\circ} \mathrm{C}$, $\mathrm{T} \sim 240 \div 280{ }^{\circ} \mathrm{C}, \mathrm{T} \sim 290 \div 300{ }^{\circ} \mathrm{C}, \mathrm{T} \sim 310 \div 380{ }^{\circ} \mathrm{C}, \mathrm{T} \geq 400{ }^{\circ} \mathrm{C}$, decrease at $\mathrm{T} \sim 150 \div 170{ }^{\circ} \mathrm{C}$, sharp growth at $\mathrm{T} \sim 180{ }^{\circ} \mathrm{C}, \mathrm{T} \sim 240{ }^{\circ} \mathrm{C}, \mathrm{T} \sim 290{ }^{\circ} \mathrm{C}, \mathrm{T} \sim 310{ }^{\circ} \mathrm{C}$, and also voltage changes at these temperatures correspond to manifestations due to the energy states $E_{i l}, E_{i 2}, E_{i 3}, E_{i 4}$. The outgoing carriers from the valence band $E v$ are capture in $E_{i l}$ and they successively move through levels that exhibit states $E_{i 2}, E_{i 3}$, $E_{i 4}$ with temperature changes (Fig. 5b). This process occurs before these levels are fill. During the

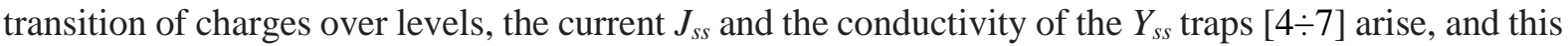
simultaneously leads to an increase in the conductivity.

\section{CONClusion}

Thus, the electrical properties depend on the granule size and location.

If the granules are arranges sequentially, the total specific resistance determined by the sum of all specific resistances. I.e, the specific resistance is relating to the granule size $\langle a\rangle$, that is, a decrease in the granule size $\langle a\rangle$ leads to an increase in $\rho$.

If the granules are parallel to each other, the charge carriers do not move from one granule to another, they are retention in traps and move along the $E_{i}$ levels in region two connected granules. During the transition of charges over levels, the current $J_{s s}$ and the conductivity of the $Y_{s s}$ traps arise, and this simultaneously leads to an increase in the conductivity. 
Thus, the comparison of the qualitative theory given in [1] with the experiments discussed here shows almost complete coincidence, the simplicity of the mechanism and its correspondence to other phenomena in granular semiconductors indicate its viability.

\section{REFERENCES}

[1] Olimov L.O., Sokhibova Z.M., Abdurakhmanov B.M.. Structure of inter grain boundaries in the granular semiconductors and the charge state

[2] Zainabidinov S.Z, Abdurakhmanov B.M, Aliev R., Olimov L.O, Mukhtarov E. Production of polycrystalline plates from silicon powder. Heliotechnics, №3, PP 79-82, 2005.

[3] Olimov L.O, Abdurakhmanov B.M, Teshaboev A. Influence of alkali metal atoms on transport of charge carriers in the between grain boundaries of polycrystalline silicon. "Material Science", №1, PP.79-82, 2014.

[4] Olimov L.O. "The grain boundaries of polycrystalline silicon: microwaves, charge states and r-n-junction". Autoreferaty of doctoral dissertation. 2016 th.

[5] Olimov L.O., Abdurakhmanov B.M., Omonboev F.L. Some Features of the Transport of Charge Carriers in the Grain Boundaries of Polycrystalline Silicon International Journal of Advanced Research in Physical Science (IJARPS) 1(6): PP 12-17, 2014.

[6] Polycrystalline semiconductors. Physical properties and applications: Per. With the English. Sub.red. Harbek G.-M., "World." 1989. 344 p.

[7] Olimov L.O. Model of the Grain Boundary in $p-n$ Structures Based on Polycrystalline Semiconductors. Applied Solar Energy, 146(2): PP 118-121, 2014.

Citation: L.O. Olimov \& Z.M. Sokhibova. "Electrical Properties of Granular Silicon”. International Journal of Research Studies in Electrical and Electronics Engineering (IJRSEEE), 5(4), pp 1-5. DOI: http://dx.doi. org/10.20431/2454-9436. 0504001

Copyright: () 2019 Authors. This is an open-access article distributed under the terms of the Creative Commons Attribution License, which permits unrestricted use, distribution, and reproduction in any medium, provided the original author and source are credited. 